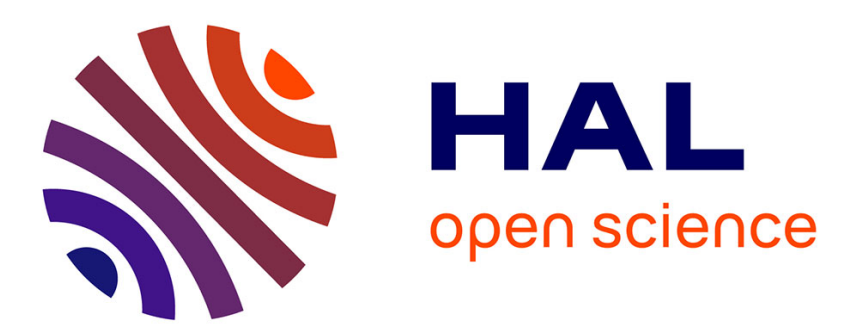

\title{
Features of the Postural Sway Signal as Indicators to Estimate and Predict Visually Induced Motion Sickness in Virtual Reality
}

\author{
Jean-Rémy Chardonnet, Mohammad Ali Mirzaei, Frédéric Merienne
}

\section{- To cite this version:}

Jean-Rémy Chardonnet, Mohammad Ali Mirzaei, Frédéric Merienne. Features of the Postural Sway Signal as Indicators to Estimate and Predict Visually Induced Motion Sickness in Virtual Reality. International Journal of Human-Computer Interaction, 2017, 33 (10), pp.771-785. 10.1080/10447318.2017.1286767 . hal-01627120

\section{HAL Id: hal-01627120 https://u-bourgogne.hal.science/hal-01627120}

Submitted on 21 Dec 2017

HAL is a multi-disciplinary open access archive for the deposit and dissemination of scientific research documents, whether they are published or not. The documents may come from teaching and research institutions in France or abroad, or from public or private research centers.
L'archive ouverte pluridisciplinaire HAL, est destinée au dépôt et à la diffusion de documents scientifiques de niveau recherche, publiés ou non, émanant des établissements d'enseignement et de recherche français ou étrangers, des laboratoires publics ou privés. 


\title{
Features of the Postural Sway Signal as Indicators to Estimate and Predict Visually Induced Motion Sickness in Virtual Reality
}

\author{
Jean-Rémy Chardonnet (1), Mohammad Ali Mirzaei, and Frédéric Mérienne \\ Le2i, Arts et Métiers, CNRS, Univ. Bourgogne Franche-Comté, HeSam, Institut Image, Chalon-sur-Saône, France
}

\begin{abstract}
Navigation in a 3D immersive virtual environment is known to be prone to visually induced motion sickness (VIMS). Several psychophysiological and behavioral methods have been used to measure the level of sickness of a user, among which is postural instability. This study investigates all the features that can be extracted from the body postural sway: area of the projection of the center of gravity (mainly considered in past studies) and its shape and the frequency components of the signal's spectrum, in order to estimate and predict the occurrence of sickness in a typical virtual reality (VR) application. After modeling and simulation of the body postural sway, an experiment on 17 subjects identified a relation between the level of sickness and the variation both in the time and frequency domains of the body sway signal. The results support and go further into detail of findings of past studies using postural instability as an efficient indicator of sickness, giving insight to better monitor VIMS in a VR application.
\end{abstract}

\section{Introduction}

It is well admitted that exposure to motion is prone to sickness, typically when being on a moving platform (e.g., a car, a train, a plane) or viewing a visual motion as stationary observers. The former case is referred to as motion sickness (MS) in the literature, while the latter is referred to as visually induced motion sickness (VIMS) (Bos, Bles, \& Groen, 2008; Oman, 1990). Sickness is characterized by physical signs of malaise such as cold sweating, belching, retching, pallor, decreased gastric tonus, and typical subjective symptoms such as headache, stomach discomfort, feeling of warmth, nausea, and eventually vomiting.

MS has been studied over decades, especially in aviation. Past research showed that adaptation and repeated exposure can minimize sickness effects (Money, 1972). However, each person is not susceptible to MS in the same way. For instance, labyrinthine-defective people, i.e., without any functioning organs of balance in the inner ears, never get sick from motion (Irwin, 1881; James, 1881; Kennedy, Graybiel, McDonough, \& Beckwith, 1968). Moreover, these patients do not suffer from visual motion, even in the absence of physical self-motion (Cheung, Howard, \& Money, 1991; Cheung, Howard, Nedzelski, \& Landolt, 1989; Johnson, Sunahara, \& Landolt, 1999). Passive persons get sicker than active persons, i.e., who control their motion themselves (Rolnick \& Lubow, 1991; Stanney \& Hash, 1998).

Based on initial research in this area, Reason and Brand suggested the so-called "sensory conflict theory" (Reason \& Brand, 1975). Bos et al. (2008) extended this theory showing that "people only get sick when there is an (apparent) change of gravity with respect to their head" (p. 48).
Virtual reality (VR), which is gathering great attention in many fields, faces the same issue as a typical task is navigation in a $3 \mathrm{D}$ immersive virtual environment (VE), thus exposing a user to visual motion. It is well known that immersive systems such as CAVEs, head-mounted displays (HMDs), and car simulators are prone to generate sickness (see Kolasinski, 1995, for a pioneering study on the topics; Darken \& Sibert, 1996; Kemeny, 2014; Kemeny, Colombet, \& Denoual, 2015), especially for users who never experienced immersion. This sickness is often referred to as cybersickness. In typical industrial applications, for instance during a project review session of a scale-one virtual building where navigation in the 3D model is required, sickness can affect decision-making and even the whole project. Different parameters are involved in VIMS, such as circular and linear vection, rod and frame effects, pseudo Coriolis and Purkinje effects, latency, foreground/background motion, the field of view, the image velocity, frame rates, and so on. Past research put effort to better understand the mechanisms laying behind VIMS occurrence, aiming at finding means to lower or even predict sickness when exposed to visual stimuli (e.g., Diels, 2008).

In this article, we focus on VIMS occurring during navigation in a $3 \mathrm{D}$ immersive VE. The main goal of this work is to provide tools to estimate and predict VIMS occurrence. A set of symptoms might appear due to a susceptibility to VIMS during or after exposure to certain dynamic visual displays. VIMS can be measured by psychological and physiological methods (Hettinger \& Riccio, 1992). The simulator sickness questionnaire (SSQ) is a well-known psychological method for measuring the extent of MS in simulators (Kennedy, 
Lane, Berbaum, \& Lilienthal, 1993a). Though the SSQ has been primarily used for aviation purposes, VR has been using this questionnaire extensively. Physiological measurement methods include heart rate variability, blood pressure (e.g., Holmes \& Griffin, 2001), electrogastrography (e.g., Himi et al., 2004), and galvanic skin reaction (e.g., Yokota, Aoki, Mizuta, Ito, \& Isu, 2005). A last method related to behavior is human postural sway (e.g., Takada, Fujikake, Miyao, \& Matsuura, 2007).

The human postural sway during upright standing is an interesting feature among the above-mentioned ones that can provide a good feedback from the user during immersion in a synthetic environment. Indeed, compared to other methods, the measurement of the human postural sway is less constraining for the user as it can be measured either using cameras or a detection balance board. The human upright standing is preserved by an involuntary physiological adjustment mechanism called the "righting reflex" (body balance function (Okawa, Tokita, Shibata, Ogawa, \& Miyata, 1995)). Sensory signals such as visual, auditory, and vestibular as well as proprioceptive signals from the skin, the muscles, and the joints trigger the function (Kaga, 1992). However, this mechanism malfunctions when exposed to $3 \mathrm{D}$ visual stimuli (Takada et al., 2007), and significant increases of the postural sway may precede the onset of MS (Stoffregen, Hettinger, Haas, Roe, \& Smart, 2000). Smart, Stoffregen, and Bardy (2002) investigated whether MS could be predicted by postural instability, exposing the participants to an optical simulation of body sway. Recent studies on animals showed that in case they have not acquired strategies to maintain their balance they may experience sickness symptoms (Riccio \& Stoffregen, 1991; Stoffregen et al., 2000). Stabilometry has been widely proposed in clinical studies, with the analysis of several parameters such as the area of sway, the total locus length of the center of gravity (COG), and the locus length per unit area (Okawa et al., 1995). Among these parameters, Takada et al. (2007) noticed that the dilation of the COG's projection area changes during exposure to stereoscopic images, more specifically, its density moves from a dense distribution to a sparse one, implying that this parameter would be an interesting feature for detecting and measuring VIMS. Past research used postural stability to evaluate the level of sickness in VR (Cobb, 1999; Kennedy \& Stanney, 1996; Murata, 2004); however, the analysis of the body sway signal in the time domain sometimes does not give enough features to detect VIMS. Moreover, most of the studies conducted were done using HMDs or large screens, whereas in this research, we use a CAVE immersive system, which arises different issues than with HMDs or large screens.

We contribute to this research by using not only the dilation of the COG's projection area (mostly considered in past studies), but also its shape and the spectrum of the body sway signal, as efficient features to better identify VIMS and monitor the level of sickness in a typical navigation task in a 3D immersive VE. We hypothesize that (1) when VIMS appears the shape of the COG's projection area changes from an ellipse to a circle as well as it dilates (H1), and (2) the frequency components of the body sway's spectrum split into two parts: frequencies corresponding to body's involuntary movements (presence of sickness) move away from the ones corresponding to body's voluntary movements (the user controls his/her body's movements) (H2).

To prove the above-mentioned hypotheses, we first review in Section 2 the theory of VIMS from a modeling perspective. Next, in Section 3, we describe the modeling of postural sway and its simulation both in the time and frequency domains to extract features to explain VIMS. We then describe in Section 4 the experiment we set up to assess the simulation and analyze the results again in both the time and frequency domains. The features obtained from the analysis will allow us to propose an implementation of a prediction system of sickness in a VR application in Section 5.

\section{Theory of VIMS}

Reason and Brand's "sensory conflict" or "sensory rearrangement" theory is the most cited theory on MS. They stated that "motion sickness is a self-inflicted maladaptation phenomenon which occurs at the onset and cessation of conditions of sensory rearrangement when the pattern of inputs from the vestibular system, other proprioceptors and vision is at variance with the stored patterns derived from recent transactions with the spatial environment" (Reason \& Brand, 1975, pp. 274-275).

A conceptual Bayesian model of this cognitive process was proposed by Oman (1990), shown in Figure 1a. The input of the model is an exogenous (externally generated) motion stimulus (e.g., a moving image), and the output generated is a sensory conflict. Though the model applies to MS without

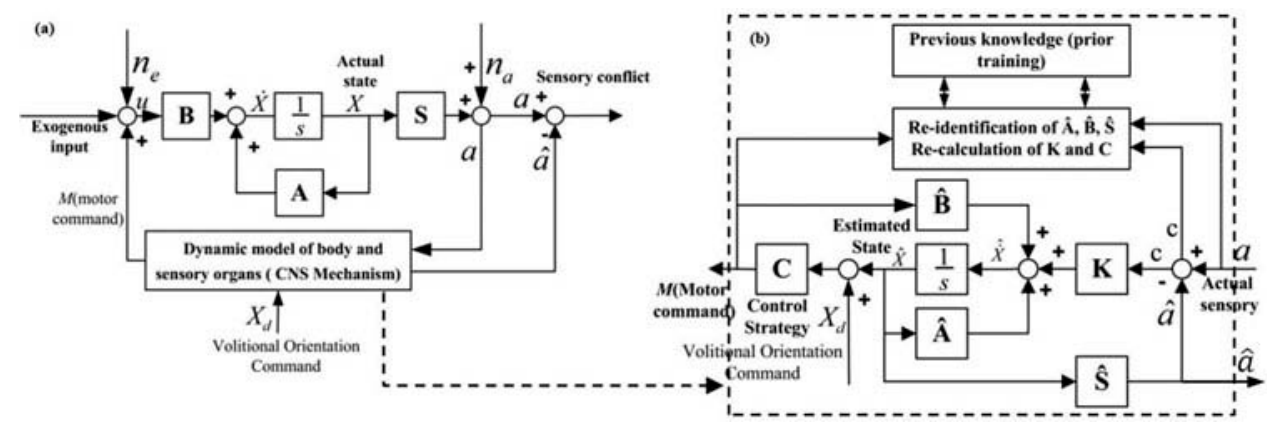

Figure 1. (a) MS model proposed by Oman (1990) for the sensory conflict theory; (b) dynamic model of the body, sensory organs and their signaling with the CNS (adapted from Oman 1990). 
detailing which motion cues matter, the visual cues in VR applications (in particular navigation scenarios) leading to cybersickness are close to those mattering for MS. A, B, and $\mathbf{S}$ are the matrices embodying the coefficients of the state differential equations for the body and the sense organ dynamics, $n_{\mathrm{e}}$ and $n_{\mathrm{a}}$ are other exogenous inputs and sensory noises, respectively. Figure $1 \mathrm{~b}$ details the central nervous system (CNS) mechanism, which plays a vital role in different mechanisms of the human body and acts here as an observer. Hatted variables of the state estimator with its matrices $\hat{\mathbf{A}}, \hat{\mathbf{B}}$, and $\mathbf{S}$ represent the neural stores of Reason's more qualitative model (Reason, 1978). The CNS continuously estimates and predicts the body orientation, and corrects the posterior estimation with a Kalman gain K (Kalman, 1960). After correction, it reidentifies $\hat{\mathbf{A}}, \hat{\mathbf{B}}$, and $\hat{\mathbf{S}}$, recalculates the Kalman gain, and updates the control strategy $\mathbf{C}$. This chain is repeated infinitely. The difference between the actual sensory a and the expected input $\hat{\mathbf{S}} \hat{\mathbf{X}}$ gives the sensory conflict vector $\mathbf{c}$ leading to sickness. When the image velocity exceeds a certain threshold, vestibular data is missing, resulting in a growth of the conflict vector and so of Kc, which provokes more sickness.

Sensory conflict can occur due to vestibular-ocular, intraocular, intra-vestibular, or ocular-vestibular conflicts with a proprioceptive pattern. The proprioceptive pattern is defined as a pattern derived from a recent transaction with a real environment. It is adjusted and the cortex is trained in the real environment by observing events, feeling the physical properties of objects, and interacting with the real environment. However, the objects and events in a VE are quite artificial with only few physical properties. Due to the lack of physical tangible properties such as gravity, force feedback, temperature, and so on, the difference between the real environment and VEs is unavoidable even though the VE is processed, visualized by very fast and high-end graphic processors, and can enable interaction with force feedback. The visual-non-vestibular difference perceived by immersion into the VE increases the amplitude of the sensory conflict vector c, which leads to strong VIMS (typically, the Kalman gain is over- or underestimated). VIMS in a synthetic environment mainly emerges due to oculo-vestibular and oculonon-vestibular conflicts. In some cases, VIMS appears due to intra-sensory conflicts (Sharples, Cobb, Moody, \& Wilson, 2008). Due to the recalculation error of the Kalman gain, the sensory motor commands are affected, thus the stance posture stability is also affected. This explains one of the reasons of choosing postural instability as a criterion for estimating and predicting VIMS. We will now model the postural sway, simulate it, then perform an experiment to correlate VIMS and features extracted from the postural sway.

\section{Postural sway modeling and simulation}

\subsection{Modeling}

The human quite upright stance is inherently unstable since it requires a large body consisting of multiple flexible segments to be kept in an upright posture with the COG located high above a relatively small base of support. Since the COG is located in front of the ankle joint, a plantar flexing torque (Loram \& Lakie, 2002; Masani, Popovic, Nakazawa, Kouzaki, \& Nozaki, 2003; Morasso \& Schieppati, 1999; Smith, 1957) and an additional active torque (Loram \& Lakie, 2002; Morasso \& Schieppati, 1999), regulated by the CNS and produced by the plantar flexors, are continuously required to prevent the body from falling.

The body dynamic and kinematic models during a quiet stance are described using an inverted pendulum (Figure 2). Figure 3 illustrates the schematic of the model in one direction of the movement, which was adapted from Masani et al. (2003). The input of the body model is the total torque exerted about the ankle joint. A proportional-differential (PD) controller, with proportional and derivative gains $K_{\mathrm{p}}$ and $K_{\mathrm{d}}$ respectively, is used to simulate the regulation of the balance performed by the CNS. A low-pass filter after the derivative gain is used to smooth the controller output signal. $\tau_{\mathrm{M}}$ is the motor command time delay, which represents the cumulative time loss due to the sensory motor information process in the CNS and the neural transmission from the CNS to the plantar flexors. $\tau_{\mathrm{E}}$ is the electromechanical response time, which represents the time difference between the moment a muscle activity signal is generated in the sensory motor and the moment the muscle reacts. $\tau_{\mathrm{F}}$ is the time delay that represents the latency recorded from the instant the sensory stimulation is provided to the foot, to the instant the sensory evoked potential is recorded in the brain. These three time constants, $\tau_{\mathrm{E}}, \tau_{\mathrm{M}}$, and $\tau_{\mathrm{F}}$, are referred to as the cognitive parameters of the model.

The motor command $M_{\mathrm{c}}$ is calculated using the COG's position and velocity information according to the following equation:

$$
M_{\mathrm{c}}(t)=-K_{\mathrm{p}} \theta\left(t-\tau_{\mathrm{F}}-\tau_{\mathrm{M}}\right)-K_{\mathrm{d}} \dot{\theta}\left(t-\tau_{\mathrm{F}}-\tau_{\mathrm{M}}\right)
$$

where $\theta$ describes the angular deviation from the upright position. After applying the Laplace transform to (1), we get:

$$
M_{\mathrm{c}}(s)=\left[-K_{\mathrm{p}}-K_{\mathrm{d}} s\right] \theta(s) \mathrm{e}^{-\left(\tau_{\mathrm{F}}+\tau_{\mathrm{M}}\right)}
$$
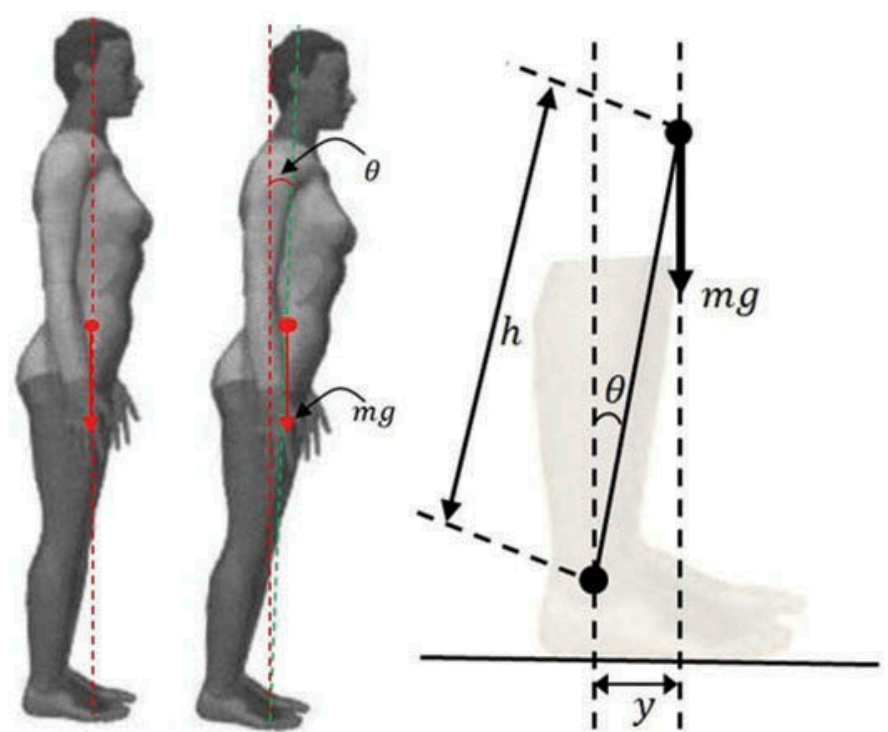

Figure 2. Dynamic model of the body sway during a quite upright stance. 


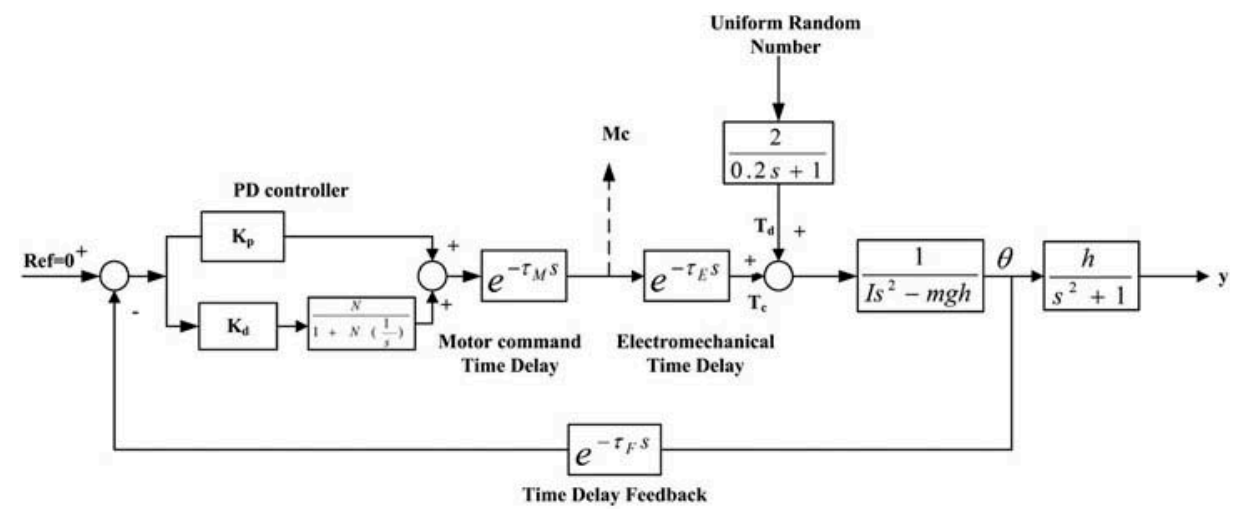

Figure 3. Inverted pendulum model and closed-loop control scheme of a quiet stance.

To show the effect of indigenous (internal from the CNS) and exogenous (external physical) forces, equation (2) can be written as:

$$
M_{\mathrm{c}}(s)=\left[K_{\mathrm{p}}+K_{\mathrm{d}} s\right] \theta(s) \mathrm{e}^{-\tau_{\mathrm{M}}} \times\left(0-\mathrm{e}^{-\tau_{\mathrm{F}}}\right)
$$

As illustrated in Figure 2, the gravity forces the body to get away from its upright equilibrium. The motion of the human body during the upright posture is rotational; therefore, the dynamic model of the body can be written as:

$$
M=I \ddot{\theta}(t)=T(t)+m g h \sin (\theta(t))-b \dot{\theta}(t)
$$

where $I$ represents the moment of inertia and $M$ denotes the total momentum acting on the body. $T=T_{\mathrm{c}}+T_{\mathrm{d}}$ represents the torque applied at the base with $T_{\mathrm{c}}$ and $T_{\mathrm{d}}$ the command torque and the disturbance torque respectively, $m g h \sin (\theta(t))$ is the influence of gravity with $m$ the mass of the body, and $-b \dot{\theta}(t)$ describes a damping effect.

The solution $\theta(t)$ to this ordinary differential equation predicts the motion of the body around the vertical axis. We assume the human body as a system without any loss and damping, just for the simplicity of the simulation and analysis (see Masani et al., 2003, for more details). Moreover, we will assume small deviation from the upright position, so that $\sin (\theta(t)) \approx \theta(t)$, which gives the linearized model:

$$
I \ddot{\theta}(t)=T(t)+m g h \theta(t)
$$

After applying the Laplace transform to (5) and assuming all the initial conditions zero, we get:

$$
T(s)=I s^{2} \theta(s)-m g h \theta(s) \Rightarrow H(s)=\frac{\theta(s)}{T(s)}=\frac{1}{I s^{2}-m g h}
$$

The model shown in Figure 3 applies to only one direction (either forward/backward (F/B) or left/right (L/R) movements); however, with different values of the cognitive parameters (PD gains and time delays), we can simulate both $\mathrm{F} / \mathrm{B}$ and $\mathrm{L} / \mathrm{R}$ movements. Under different cognitive parameters reconfigurations, the model can successfully describe body movements.

\subsection{Simulation}

Based on the classic control theory (Khalil, 2002) for any closeloop control process, there exist three states: stable, marginally
Table 1

\begin{tabular}{lcccccc}
\hline & \multicolumn{6}{c}{ Parameters } \\
\cline { 2 - 7 } & $\mathrm{T}_{\mathrm{F}}(\mathrm{ms})$ & $\mathrm{T}_{\mathrm{E}}(\mathrm{ms})$ & $\mathrm{T}_{\mathrm{M}}(\mathrm{ms})$ & $\mathrm{K}_{\mathrm{p}}$ & $\mathrm{K}_{\mathrm{d}}$ & $\mathrm{N}$ \\
\hline Range & $35.1-40.1$ & $10.54-11.5$ & $25-215$ & $50-3000$ & $50-2000$ & $5-2000$ \\
S.L/R & 40 & 11 & 135 & 680 & 320 & 15 \\
S.F/B & 40 & 11 & 135 & 750 & 350 & 20 \\
MS. L/R & 40 & 11 & 175 & 680 & 350 & 9 \\
MS. F/B & 40 & 11 & 175 & 700 & 380 & 10 \\
\hline
\end{tabular}

stable, and unstable. Stability during a stance posture indicates that the body stays upright with very tiny movements. In this state, larger movements mean only voluntary movements (the person controls his/her body movements). On the contrary, the body tends to fall down when it is unstable. During marginally stable, the body tends to become unstable while the CNS tries to keep it upright by sending commands to different joints using the righting reflex function. Consequently, the body physically fluctuates around its vertical axis and the CNS alternates between stable and unstable states. In other words, the movements of the body are mostly involuntary movements (the person can hardly control his/her body movements). Since the upright postural sway is important in this study, only the stable and marginally stable states will be considered.

The controller and the cognitive (CNS) parameters are set to the values shown in Table 1. Note that the exact value of the motor command time delay, i.e., the time needed for the sensory motor information to be processed in the CNS, is unknown. The different constants of the body transfer function are calculated for a male adult as found by Masani et al. (2003) $\left(m=76 \mathrm{~kg}, I=66 \mathrm{~kg} \cdot \mathrm{m}^{2}\right.$, and $\left.h=0.87 \mathrm{~m}\right)$. Simulation was performed in MATLAB Simulink for $20 \mathrm{~s}$ and the sampling frequency was set to $100 \mathrm{~Hz}$ (MathWorks, 2015).

\section{Time-domain analysis}

The resulting simulation signals of $\mathrm{F} / \mathrm{B}$ and $\mathrm{L} / \mathrm{R}$ movements for marginally stable and stable states are shown in Figure 4a and $b$, respectively. As shown, the amplitude of the signals is higher for the marginally stable state compared to the stable state. Besides, the amplitude of the signals for the marginally stable state at the beginning is low and increases exponentially by time, while the external input Ref $=0$, in Figure 3, is always zero during the entire simulation time. 
To better highlight the difference between the marginally stable and the stable states, the F/B and L/R signals of Figure $4 a$ and $b$ are projected onto the $\mathrm{XY}$ plane, as shown in Figure 5. The area and the shape of the COG's projection are then considered as features to compare the body sway in the stable and marginally stable states. By definition (TechnoConcept, 2007), the COG's projection area is defined as an optimum ellipse surrounding $90 \%$ of the points. The confidential interval is calculated from the scatter center, not from the origin of the coordinates.

As seen, the body does not move widely in the stable state, hence the COG's projection area (Figure 5b) is quite dense and smaller than in the marginally stable state. Moreover its shape is elliptical. In the marginally stable state, the body movement is larger, the corresponding area dilates significantly with a shape tending to a circle (Figure 5a).

This observation gives us one feature for the estimation of VIMS, as it will be shown in the user studies.

\section{Frequency-domain analysis}

The $L / R$ and F/B sway signals previously generated are converted into a spectrum-frequency representation using the fast Fourier transform (FFT) in order to analyze and interpret the signals in the frequency domain, and disclose aspects that are not visible in the time-domain representation. Recall that in the marginally stable state, mostly involuntary movements occur, while in the stable state, only voluntary movements occur. Bos (2003) showed the switch between voluntary and involuntary movements can be set at $1 \mathrm{~Hz}$. Though this $1 \mathrm{~Hz}$ switch can depend on individual differences, we considered this frequency as a good estimation. In the rest of the article, we note LF (low frequency) the frequency components below $1 \mathrm{~Hz}$ (associated with voluntary movements), while HF (high frequency) denotes the frequency components above $1 \mathrm{~Hz}$ (associated with involuntary movements).

Figure 6 shows the spectrum of the F/B signal of Figure 4 for the marginally stable state (Figure $6 a$ ) and the stable state (Figure $6 \mathrm{~b}$ ). Since the F/B and L/R signals are similar both in characteristics and in shape, only the F/B signal will be analyzed in this section for clarity.

As shown in Figure 6a, the spectrum of the marginally stable F/ B sway has two components, regardless of the stimulus causing the sway, external or internal. One component stretches beyond $1 \mathrm{~Hz}$, i.e., this component is associated to involuntary movements, while the other component remains below $1 \mathrm{~Hz}$, i.e., this component is associated to voluntary movements. From a VIMS modeling perspective, the malfunction of the CNS or an inaccurate estimation of the body state leads to a growing sensory conflict vector and so increased sickness.

Under a completely stable state (Figure 6b), there are only LF components, i.e., the body stays upright with minimum sway and only voluntary movements occur. Voluntary movement is slow, thus its spectrum has frequency components in a bandwidth limited to $0-0.5 \mathrm{~Hz}$.

This observation gives us a second feature for the estimation of VIMS, as it will be shown in the user studies. Especially, we will correlate these results with the sickness scores obtained by the questionnaires.
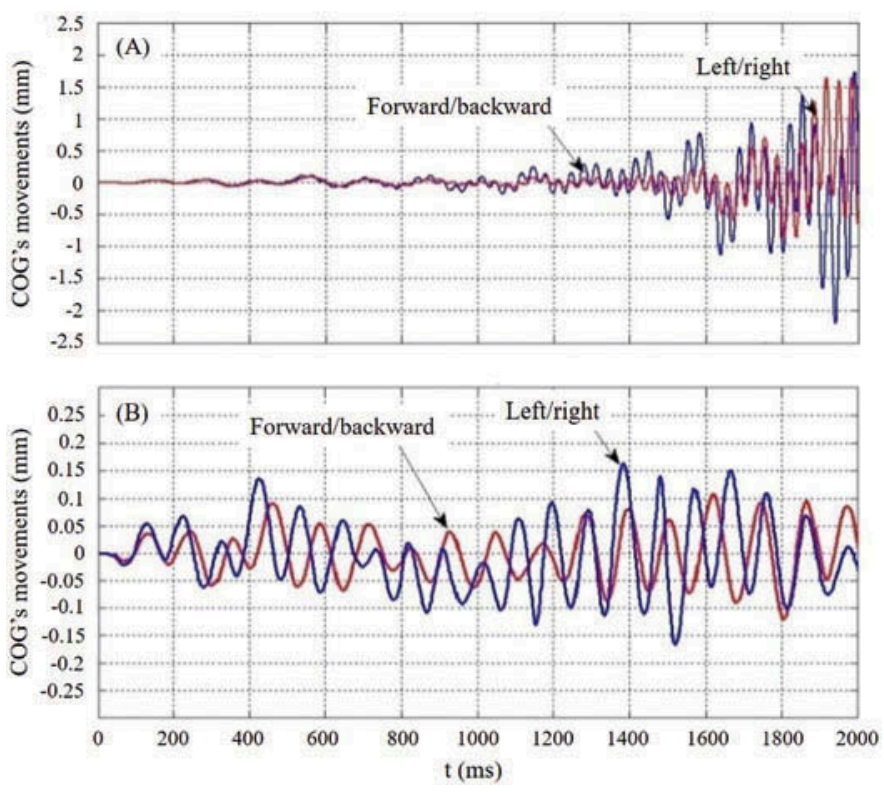

Figure 4. Forward/backward and left/right signals for (a) a marginally stable state and a (b) stable state.

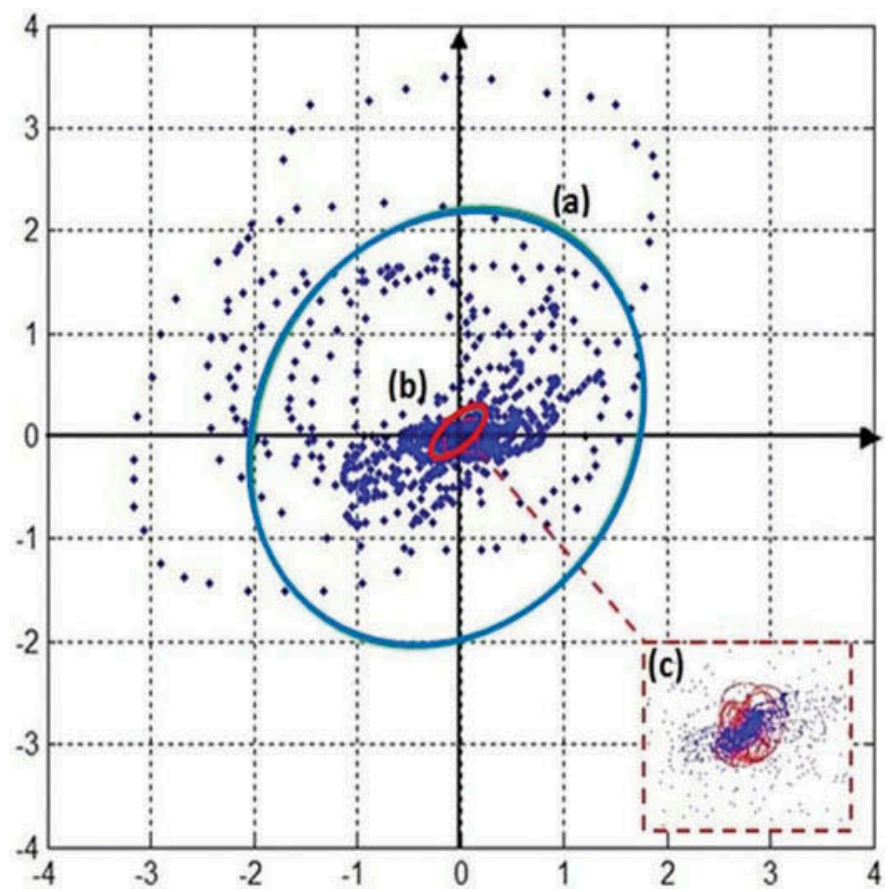

Figure 5. COG area created by projecting the forward/backward and left/right corresponding values onto the XY plane: (a) points associated with the marginally stable state, (b) points associated with the stable state, and (c) zoom in to show detail of the stable state points that are hidden under the marginally stable state points.

\section{User study}

We designed an experiment to assess the simulation results and derive features for estimating and predicting VIMS during navigation in a VR application. Recall the hypotheses we made in the introduction: 

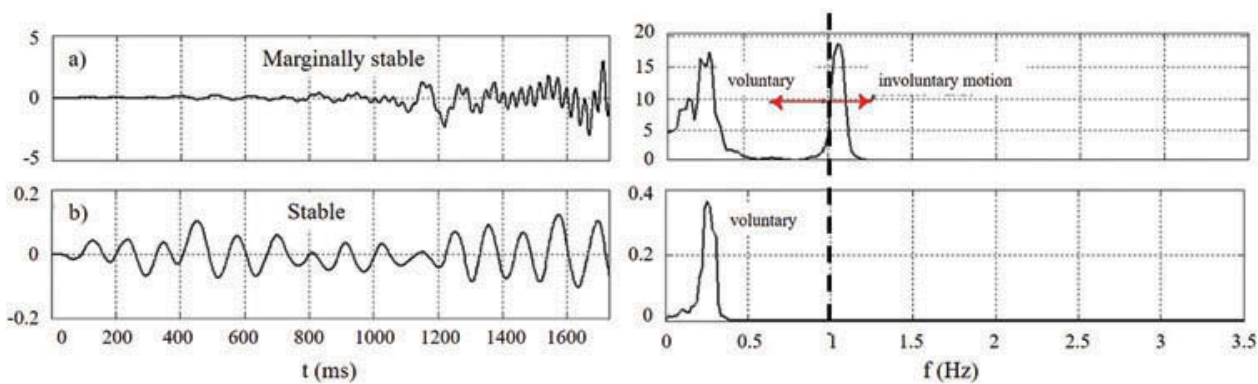

Figure 6. F/B postural sway signal and its spectrum for the (a) marginally stable and (b) stable states.

- [H1] When VIMS appears, the shape of the COG's projection area changes from an ellipse to a circle as well as it dilates.

- $[\mathrm{H} 2]$ The frequency components of the body sway's spectrum split into two parts: frequencies corresponding to body's involuntary movements (presence of sickness) move away from the ones corresponding to body's voluntary movements (the user controls his/her body's movements).

The simulation validates these hypotheses. Now we will correlate simulation with user studies.

\subsection{Navigation mechanism}

To navigate in a 3D VE, we use a so-called Flystick device. The overall navigation set-up is depicted in Figure 7. The Flystick has five buttons, one joy-stick handle, a laser-based optic tracker for both position and orientation tracking, and a trigger button, as shown in Figure 7. To move forward, the user pushes the joy-stick handle toward the desired direction, whereas rotation is done by pushing the joy-stick either to the left or the right, or by pointing the Flystick in the desired direction. Note that the position and orientation of the Flystick is not correlated to that of the user, i.e., when the user pushes the joy-stick handle toward the direction of the device, he/she moves toward that direction, independently of whether the user is looking (or his/her head is oriented) at that direction. In this study, we focused only on translational movements to avoid any biases in the measures. To start and stop navigation, the user presses the "start" and "stop" buttons, respectively. The "+" and "-" buttons shown in Figure 7 are not used.

\subsection{Experiment equipment}

The experiments were conducted in a CAVE immersive system. Our CAVE system consists of four $3 \times 3 \times 3$ m walls with two $1400 \times 1050$ px resolution projectors per wall for stereoscopic vision, an infrared-based tracking system to track the user's location in the CAVE and in the VE, and the Flystick device, as depicted schematically in Figure 7.

A custom software development platform, called iiVR and written in $\mathrm{C}++$, was developed to manage the connections within the whole VR equipment (display system, infrared cameras, hardware resources, and network of navigation/interaction devices). Rendering of the 3D virtual scenes is done using OpenSceneGraph on top of OpenGL and the properties of the VE are incorporated in the model. Using MPI and four NVidia Quadroplex GPUs, the generated model is projected at $60 \mathrm{fps}$ in stereoscopic view in the CAVE system on all walls. JavaScript is used to wrap all the C++ functions, facilitating VR applications development.

The Flystick is connected via Virtual Reality Peripheral Network (VRPN) to iiVR. The overall latency is around $40 \mathrm{~ms}$.

All data from the user studies are imported via VRPN to MATLAB and analyzed using MATLAB scripting language, Simulink, and data processing toolboxes.

We measure the participants' COG using a Techno Concept balance board (TechnoConcept, 2007). The sensor can measure $\mathrm{L} / \mathrm{R}$ and $\mathrm{F} / \mathrm{B}$ signals in real time and calculate up to 13 parameters with high accuracy compared to other balance boards such as the so-called WiiFit board.

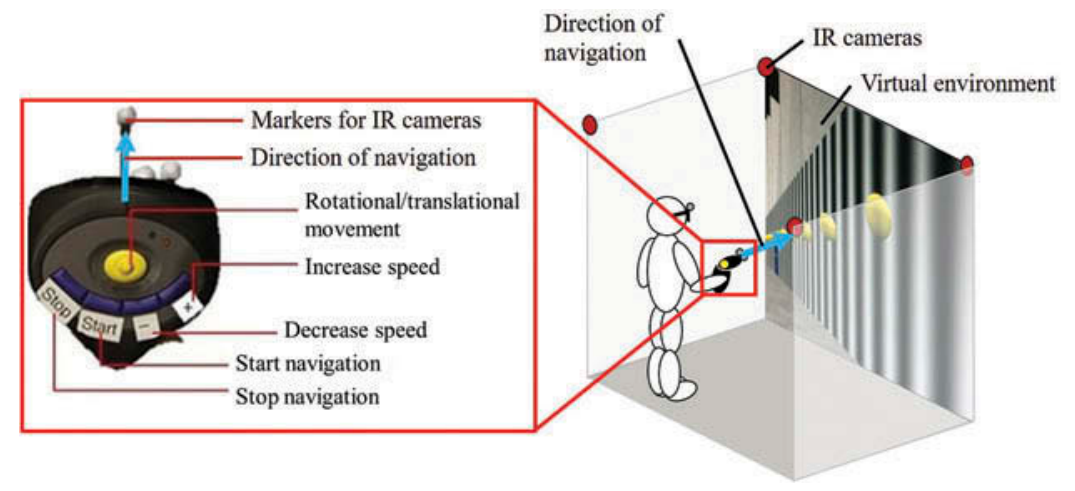

Figure 7. Setup of the whole VR navigation system with a focus on the Flystick device. 


\subsection{Participants}

Seventeen subjects ( 13 males and 4 females: $31.58 \pm 12.69$ years, $74.65 \pm 15.22 \mathrm{~kg}$ ) from the university participated in the experiment. There was a briefing to give enough information about the test procedure and possible risks before each experiment individually. All the subjects participated voluntarily (no compensation) to the experiments. A pre-exposure questionnaire (Q1) was filled by each subject to evaluate their health condition and get a better insight of their background in the usage of computers (see Appendix). From this questionnaire, no subject reported any health issues for the participation in the experiment.

\subsection{Experiment procedure}

The procedure was designed as follows:

(1) A pre-exposure questionnaire (Q1) is filled by each participant.

(2) Since the participants need to know how to navigate with the Flystick, each participant has a training period $(2 \mathrm{~min})$ before exposure to visual stimuli (base line).

(3) The COG of each participant is recorded for $t_{1}=30 \mathrm{~s}$ (pre-exposure measurement).

(4) The participants navigate along a path (shown in Figure 8 , the participant follows a row of virtual yellow balls along a wall with a specific pattern that stimulates VIMS) for $t_{2} \mathrm{~s}$ at a speed of $2 \mathrm{~m} \mathrm{~s}^{-1}$ (note that this speed is higher than a normal walking speed; however, we choose this speed to provoke VIMS more easily).

(5) The COG signal is recorded immediately after navigation for $t_{1} \mathrm{~s}$ (post-exposure measurement). In this step, the participants are removed from the CAVE. They stand still on the balance board while looking at a fixed point displayed on a wall.

(6) SSQ (Q2) is filled by each participant to calculate the sickness score. Here we do not use exactly the same SSQ calculation method as the one of Kennedy et al. (1993a): we added each rated score of each symptom for each symptom cluster, multiplied each cluster score by their specific weight factor given by Kennedy et al., and summed the three subscores then multiplied by 3.74. This leads to SSQ total score values that can be higher than 300 (this value being defined by Kennedy et al. (2003)).

(7) Steps 4-6 are repeated seven times.

The whole procedure is summarized in a timeline depicted in Figure 9.

Note that before starting the experiment we asked orally the participants whether they already felt any symptoms indicated in the SSQ. No participant reported any symptoms before the experiment, meaning they were in their usual state of fitness.

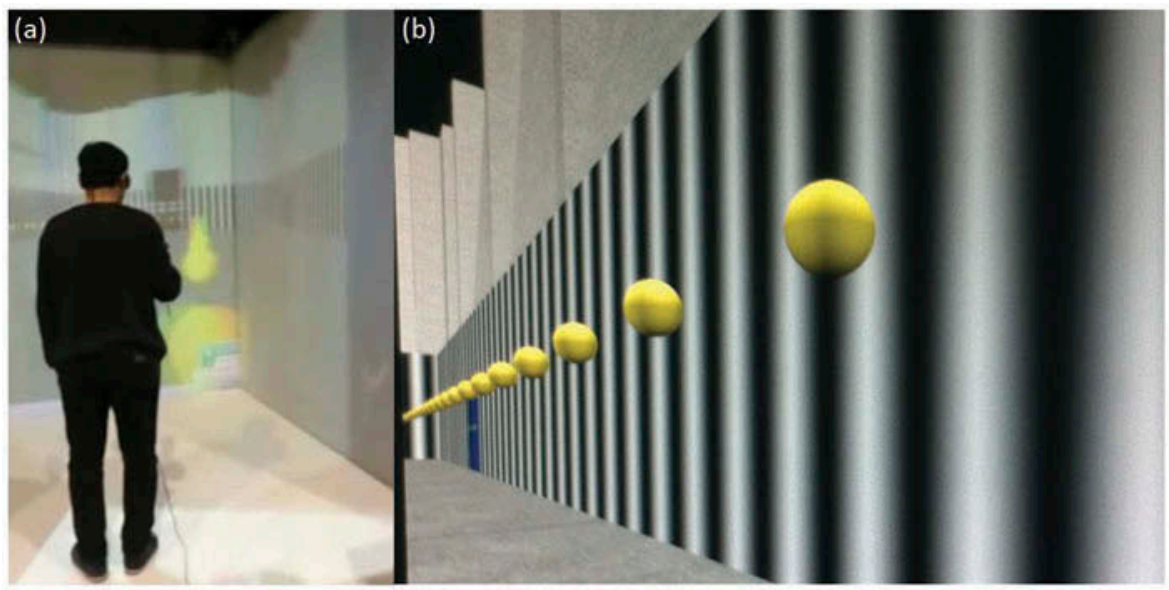

Figure 8. (a) Experiment inside the CAVE and (b) path indicator and environment pattern.

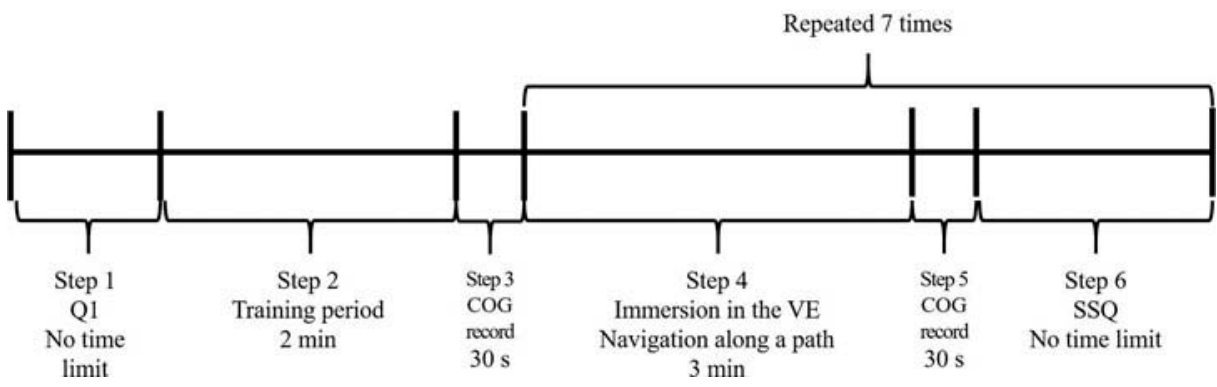

Figure 9. Timeline of the experiment. 


\section{Results}

As for simulation, the data were analyzed both in the time and frequency domains. We then correlated the results with the sickness scores given by the SSQs.

The experiment has been carried out for $t=50 \pm 20 \mathrm{~min}$ ( $3 \mathrm{~min}$ in average to complete navigation per step, the time variation is mostly due to the time needed by the participants to complete the questionnaires). The $\mathrm{F} / \mathrm{B}$ and $\mathrm{L} / \mathrm{R}$ signals are recorded directly by the COG sensor, then the parameters are computed by the interfacing software (see the Techno Concept manual for further details (TechnoConcept, 2007)).

At the end of the experiment, 8 participants out of 17 reported being quite sick (they also mentioned it verbally), but none of them stopped during the experiment.

\subsection{Time-domain analysis}

Figure 10 shows an example of a recorded F/B (red) and L/R (blue) COG's signal for pre- (light colors) and post-exposures (strong colors) for one participant. As seen, though the postexposure signals seem overall larger than the pre-exposure ones, the comparison of these two signals is difficult in the time representation without any feature extraction, on the contrary of simulation: in simulation, because ideal conditions are provided (no noise and no side effect), the signals are much clearer, thus it is much easier to extract desired features. To solve this issue, we first look at the 13 parameters from the COG sensor that are calculated from the pre- and post-exposure measurements and averaged over all the participants. We identify the most critical parameters by looking at the difference between pre- and post-exposure measurements.

Table 2 shows the 13 parameters obtained from the COG sensor. From the statistical analysis on each parameter using a one-way repeated measures ANOVA, we observed that 6 out of the 13 parameters underwent significant variations: Area $(F$ $(1,16)=198.6, p<.005)$, Length $(F(1,16)=154.9, p<.003)$, Lng. $\mathrm{L} / \mathrm{R}(F(1,16)=105.2, p<.001)$, Lng. Fr./Bk. $(F$ $(1,16)=149.5, p<.001)$, S Var. $(F(1,16)=98.23, p<.001)$, Slope $(F(1,16)=91.23, p<.01)$. The other parameters either did not undergo significant variations or did not follow a specific trend. Therefore, they have not been considered further in this study. Among all the parameters that underwent significant variation, we select especially the area parameter of the COG, considering that past research also focused on this feature to detect VIMS, as mentioned earlier.

Our measurements are done at eight points during the test; however, only three instances are more important in this study: at the beginning of the experiment (pre-exposure), at the sickness onset, and at the end of the experiment (post-exposure).

To the best of our knowledge, very little is known about the time of the sickness onset; however, in our study, we will define it
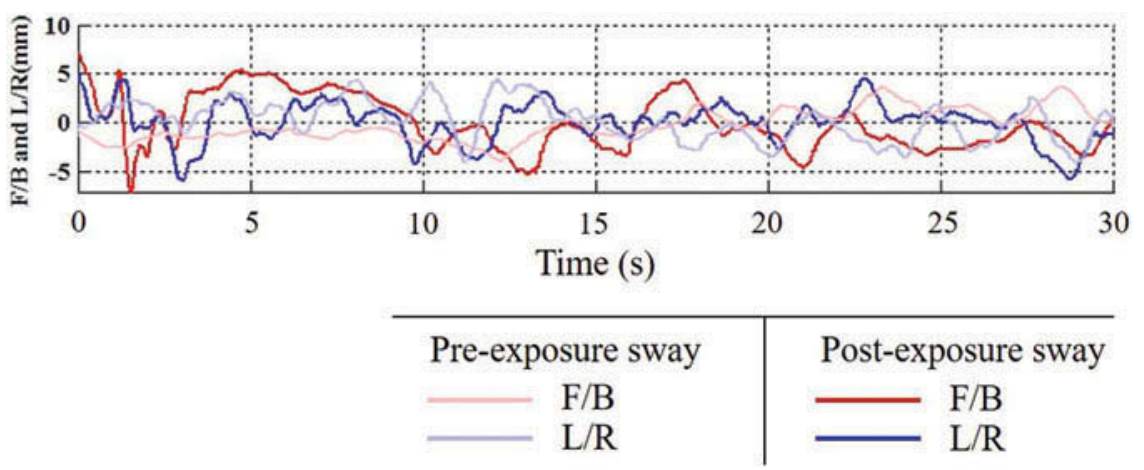

Figure 10. Example of F/B and L/R sways signals at pre- and post-exposures.

Table 2.

\begin{tabular}{|c|c|c|c|c|c|}
\hline \multirow[b]{2}{*}{ Parameters } & \multicolumn{5}{|c|}{ COG sensor data } \\
\hline & Abbreviation & Pre-exp & Post-exp & Variation & $p$-value \\
\hline Area $\left(\mathrm{mm}^{2}\right)$ & Area & 29 & 553.4 & 524.4 & .0035 \\
\hline Locus length (mm) & Length & 163 & 489.3 & 326.3 & .0023 \\
\hline Locus length Left/Right (mm) & Lng. L/R & 59.9 & 195.5 & 135.6 & .0001 \\
\hline Locus length of Forward/Backward (mm) & Lng. Fr./Bk & 127.5 & 436.9 & 309.4 & .00054 \\
\hline Speed variance $\left(\mathrm{mm} \cdot \mathrm{s}^{-1}\right)$ & S Var. & 10.7 & 188.1 & 177.4 & .00017 \\
\hline Slope & Slope & 43 & 129.3 & 86.3 & .0075 \\
\hline Forward/Backward average & Fr./Bk. Avg & -26.2 & 3 & 29.2 & .46 \\
\hline Left/Right average & L/R Avg & -8.8 & 59.8 & 68.6 & .58 \\
\hline Normal amplitude at $0.2 \mathrm{~Hz}$ Left/Right & AN02 L/R & 1.1 & 26.4 & 25.3 & .17 \\
\hline Normal amplitude at $0.2 \mathrm{~Hz}$ Forward/Backward & AN02 Fr./Bk. & 2.1 & 29.5 & 27.4 & .96 \\
\hline Length function of the surface & LFS & 0.4 & 0.9 & 0.5 & .64 \\
\hline Sway velocity Forward/Backward $\left(\mathrm{mm} . \mathrm{s}^{-1}\right)$ & SV Fr./Bk. & -17.2 & 8.2 & 25.4 & .31 \\
\hline Speed average $\left(\mathrm{mm} \cdot \mathrm{s}^{-1}\right)$ & S Avg. & 5.3 & 15.6 & 10.3 & .08 \\
\hline
\end{tabular}


as the moment where sudden change of the SSQ scores is observed between two consecutive steps of navigation, i.e., the participant starts feeling sick. This change can be calculated by introducing two indexes: a susceptibility index $S$ and an average sickness index $A S$ defined by:

$$
S=\left|\frac{\mathrm{SSQ}_{i-1}-\mathrm{SSQ}_{i}}{t}\right|, \quad A S=\frac{\sum_{i=1}^{m} \mathrm{SSQ}_{i}}{m}
$$

where $\mathrm{SSQ}_{i}$ is the sickness total score calculated from the SSQ at the end of the $i$ th step, $t$ is the time the participants spent to complete the current step, and $m$ is the number of steps that have already been completed. For example, suppose $\mathrm{SSQ}_{1}=125$, $\mathrm{SSQ}_{2}=150, \mathrm{SSQ}_{3}=300, t_{1}=3 \mathrm{~min}, t_{2}=2.5 \mathrm{~min}$ are reported, then $S_{1}=|(150-125) / 3|=8.3, S_{2}=|(300-150) / 2.5|=60, A S_{1}=275 /$ $2=137.5, A S_{2}=575 / 3=191.6$. It can be clearly seen that some event happened in step 2 because $S$ and AS highly increased.

The COG's projection area associated with these three instances, for one participant, is depicted in Figure 11. The area for each instance is superimposed to highlight the difference between them. Then, as in simulation, we define for each instance the optimum ellipse surrounding $90 \%$ of the points as the shape of the COG's projection area. From Figure 11, we can observe that the post-exposure area has a larger distribution with an almost circular shape compared to the pre-exposure area and the sickness onset area where the shape is elliptical.

We plotted in Figure 12 the schematic evolution of the shape of the COG's projection area step by step for two participants to explain how the geometrical shape of the COG's projection area varies with time. For the first participant, the area has an elliptical shape at the beginning the experiment $\left(\right.$ Area $\left.=234.38 \mathrm{~mm}^{2}\right)$, keeps elliptical till the seventh step (Area $=423.13 \mathrm{~mm}^{2}$ ), then the shape switches from an ellipse to a circle (Area $\left.=553.41 \mathrm{~mm}^{2}\right)$, indicating the participant's body sway becomes marginally stable. Therefore, following our observations in simulation in the section "TimeDomain Analysis", we can define the sickness onset also as the moment where a change in the area's shape occurs. For the second participant, the same phenomenon occurs at the fifth step. In the meantime, the area increases at each step for both participants. Obviously, as human bodies are not radially symmetrical, it is not possible to switch from an ellipse to a perfect circle; however, we observed that the ellipses tended to circles at sickness onset.
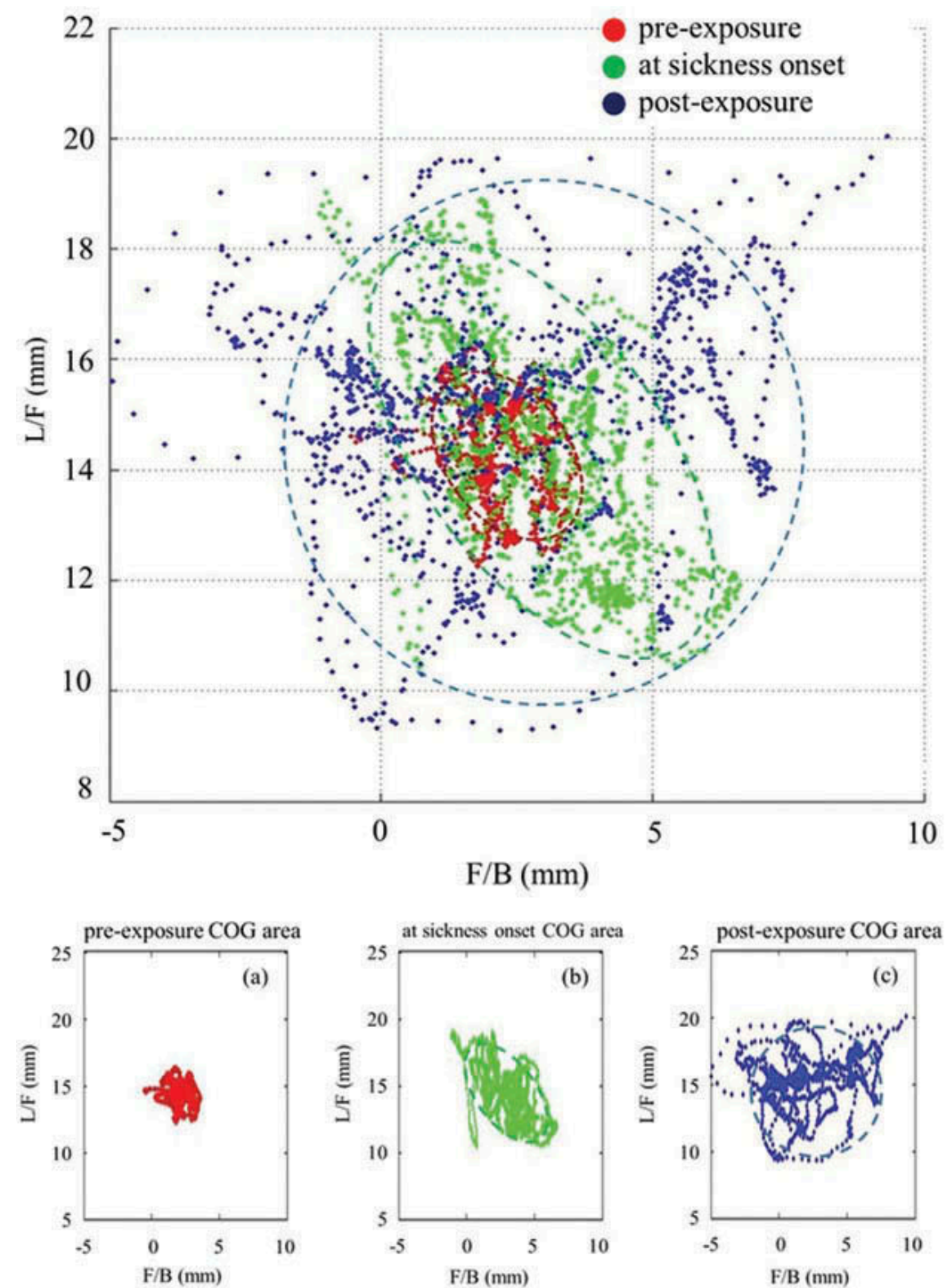

Figure 11. Variation of the COG area and the shape during the experiment, at pre-exposure (a), at sickness onset (b), and at post-exposure (c). 


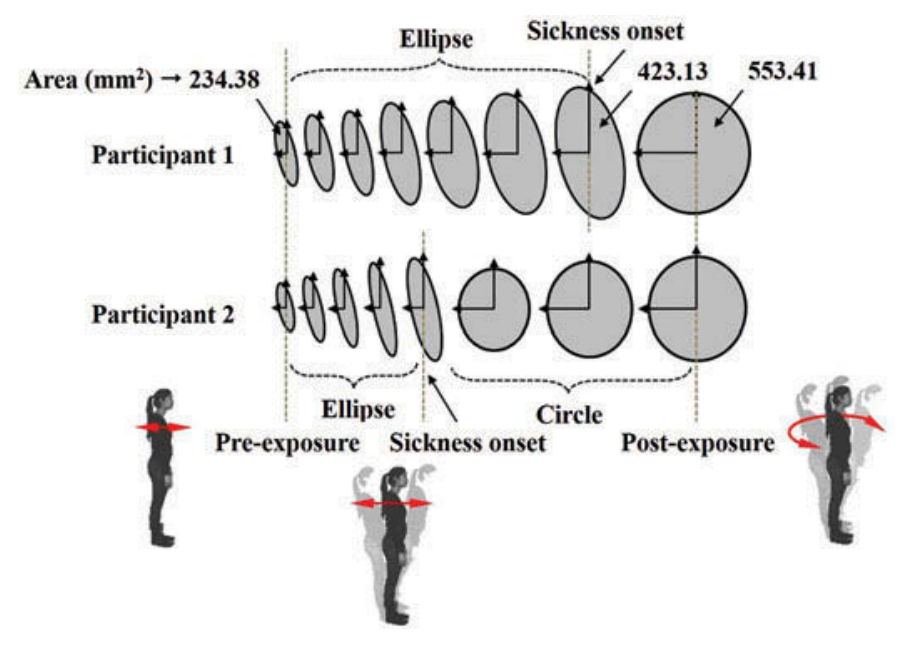

Figure 12. Shape variation of the COG's projection area during the experiment for two participants.

To be sure the variation of area and shape is due to an increase of sickness, we correlate it with the level of sickness given by the sickness scores from the SSQs. We compute for each participant the difference between the three areas, which we will denote $\Delta A_{1}=A_{S O}-A_{\text {pre }}$ between sickness onset and pre-exposure, $\Delta A_{2}=A_{\text {post }}-A_{\text {pre }}$ between post- and pre-exposures, and $\Delta A_{3}=A_{\text {post }}-A_{S O}$ between post-exposure and sickness onset. Equivalently, we compute the difference between the sickness scores from the SSQs at these three instances, which we will denote $S S Q_{1}=S S Q_{S O}-S S Q_{p r e}$ between sickness onset and pre- exposure, $S S Q_{2}=S S Q_{\text {post }}-S S Q_{\text {pre }}$ between post- and pre-exposures, and $S S Q_{3}=S S Q_{\text {post }}-S S Q_{S O}$ between post-exposure and sickness onset.

Figure 13 shows the correlation between $S S Q_{1}$ and $\Delta A_{1}, S S Q_{2}$ and $\Delta A_{2}, S S Q_{3}$ and $\Delta A_{3}$ respectively for all the participants. As shown, there is a strong correlation between $S_{S Q} Q_{1}(M=184.02$, $S D=19.83)$ and $\Delta A_{1}(M=198.13, S D=10.898): r=.96$, between $S_{S Q}(M=294.89, S D=15.3)$ and $\Delta A_{2}(M=383.19, S D=18.97)$ : $r=.97$, and between $S Q_{3}(M=111.8, S D=7.7)$ and $\Delta A_{3}$ $(M=185.35, S D=7.44): r=.98$.

We also correlated the shape of the COG's projection area with the level of sickness. An ellipse can be recognized from a circle using the ratio $R=r_{2} / r_{1}$, where $r_{1}$ and $r_{2}$ represent the short and long radii. If $r_{2}=r_{1}$, the shape is a circle, otherwise it is an ellipse. $R$ and the SSQ score are calculated at sickness onset (SO), where the change of shape occurs, and just one step after $(\mathrm{SO}+1)$, to highlight the difference of shape. Then, the difference between the two ratios $\Delta R=R_{S O+1}-R_{S O}$ and between the two SSQ scores $S S Q=S S Q_{S O+1}-S S Q_{S O}$ are extracted for each participant. Statistical analysis across the subjects shows that the ratio difference $\Delta R(M=0.41$, $S D=0.17)$ and the SSQ difference $(M=112.4, S D=8.98)$ are highly correlated $(r=.95)$ as plotted in Figure 14 .

As a result, time-domain analysis of the body sway signal shows that when a user navigates in an immersive VE and becomes sick, (1) the distribution of the COG's projection becomes less dense with a larger spatial distribution, (2) the shape of its area switches from an ellipse to a circle, (3) the area increases, with an increase of the level of sickness. We (a)

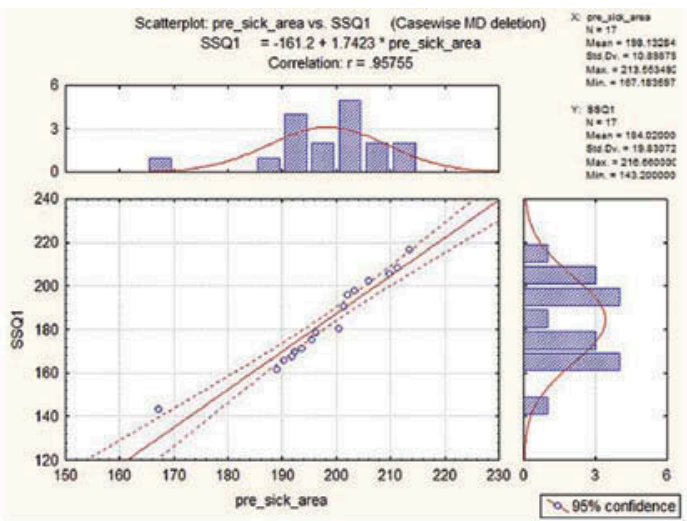

(b)

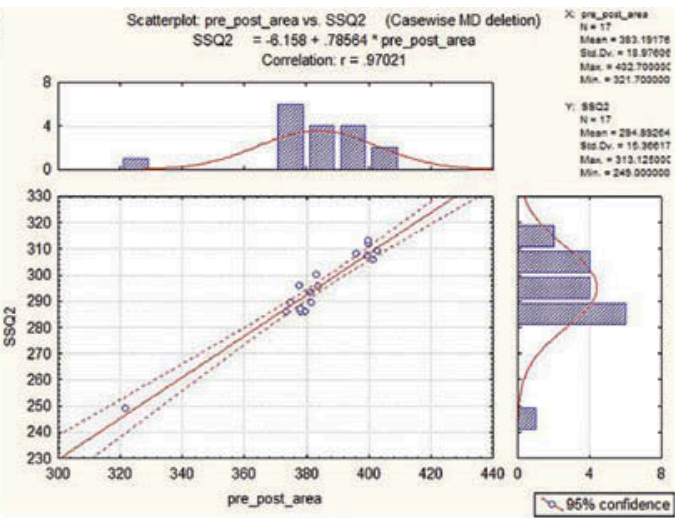

(c)

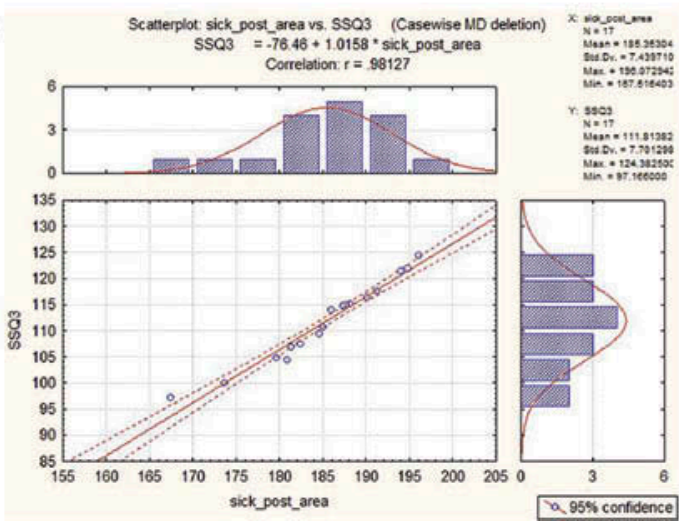

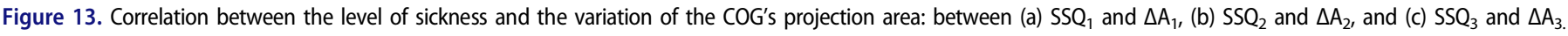




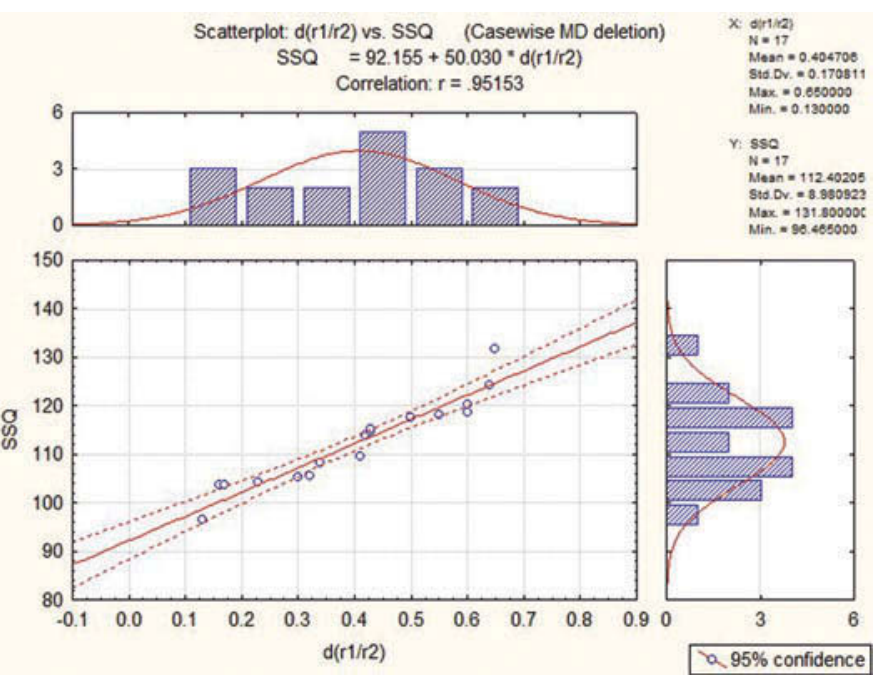

Figure 14. Correlation between the COG's projection shape and the sickness score.

therefore assessed the simulation and the first hypothesis. The COG's projection area as well as its shape is an efficient indicator of the level of sickness of the user.

\subsection{Frequency-domain analysis}

As in simulation, the $\mathrm{F} / \mathrm{B}$ and $\mathrm{L} / \mathrm{R}$ sway signals of all the participants of the experiment are transformed into the frequency space using the FFT. In this section, only the analysis for the F/B signal will be presented for clarity of the article; however, the analysis was conducted considering both F/B and $\mathrm{L} / \mathrm{R}$ signals. The frequency components of the F/B signal corresponding to one participant are illustrated in Figure 15 at the same three instances: at pre-exposure, at sickness onset, and at post-exposure. A curve is fitted over each sample to make them visually recognizable and distinguishable from one to another.

Recall that frequency components that are below $1 \mathrm{~Hz}$ correspond to voluntary movements (LF components, as defined in "Frequency-Domain Analysis") while those above $1 \mathrm{~Hz}$ correspond to involuntary movements (HF components). The vertical red dotted line in Figure 15 indicates the first frequency component of the voluntary movements (this component for the three instances expand approximately on the same interval). The green and blue dotted lines show the first component of the involuntary movements at sickness onset and at postexposure, respectively.

The pre-exposure F/B signal (in red color) has two frequency components: $0.0-0.36 \mathrm{~Hz}$ and $0.43-0.93 \mathrm{~Hz}$, which are below $1 \mathrm{~Hz}$, thus we classify them as LF components, meaning they are related to voluntary movements (no sickness felt by the participant). The F/B signal associated with sickness onset (in green color) has two LF components, 0.0$0.36 \mathrm{~Hz}$ and $0.5-1.2 \mathrm{~Hz}$, and one $\mathrm{HF}$ component, 1.18$2.63 \mathrm{~Hz}$. According to the previous discussion in Section 3.2.2, the user starts feeling sickness as he cannot fully control his body movements. The post-exposure F/B signal (in blue color) has three LF components, 0.0-0.4 Hz, 0.45$0.79 \mathrm{~Hz}$, and $0.85-1.2 \mathrm{~Hz}$, and one HF component, 1.75$2.85 \mathrm{~Hz}$, that is further away than the HF component of the sickness onset signal. Note that for one participant who reported being quite sick at the end of the experiment we even observed $\mathrm{HF}$ components beyond $5 \mathrm{~Hz}$.

We computed for each participant the difference between the HF component at sickness onset and the first LF component at pre-exposure $\Delta \mathrm{f}_{1}=\mathrm{HF}_{\mathrm{SO}}-\mathrm{LF}_{\text {pre }}$ (see Figure 15), between the HF component at post-exposure and the first LF component at pre-exposure $\Delta \mathrm{f}_{2}=\mathrm{HF}_{\text {post }}-\mathrm{LF}_{\text {pre }}$, and between the $\mathrm{HF}$ component at post-exposure and the $\mathrm{HF}$ component at sickness onset $\Delta \mathrm{f}_{3}=\mathrm{HF}_{\text {post }}-\mathrm{HF}_{\mathrm{SO}}$. As in the time-domain analysis, to be sure the variation of the frequency components, especially the fact that after sickness onset an HF component appears and moves away from $1 \mathrm{~Hz}$, i.e., the difference between HF and LF components increases, is due to an increase of sickness, we correlate it with the level of sickness given by the sickness scores from the SSQs.

Figure 16 shows the correlation between $S_{S Q}$ and $\Delta f_{1}$, $\mathrm{SSQ}_{2}$ and $\Delta \mathrm{f}_{2}, \mathrm{SSQ}_{3}$ and $\Delta \mathrm{f}_{3}$, respectively, for all the participants. As shown, there is a strong correlation between $\mathrm{SSQ}_{1}$ and $\Delta \mathrm{f}_{1}(M=1.66, S D=0.10): r=.96$, between $\mathrm{SSQ}_{2}$ and $\Delta \mathrm{f}_{2}$ $(M=2.65, S D=0.28): r=.97$, and between $S_{S Q}$ and $\Delta \mathrm{f}_{3}$ $(M=0.99, S D=0.34): r=.97$.

As a result, when the difference between the LF and HF components of a postural sway increases $(M=0.99 \mathrm{~Hz} \rightarrow$ $M=2.63 \mathrm{~Hz})$, the VIMS score grows $(M=111.81 \rightarrow$

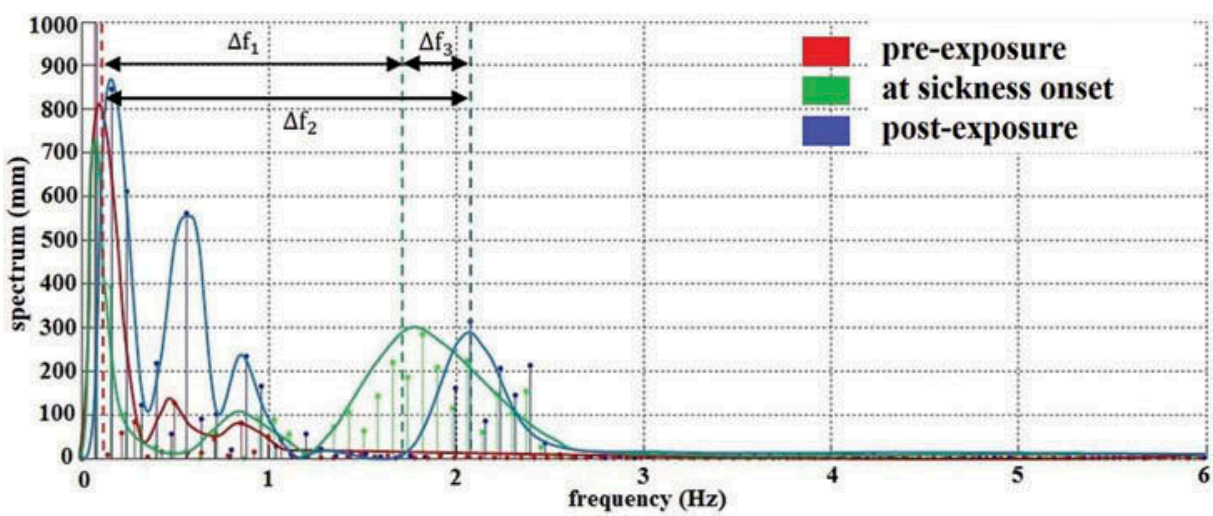

Figure 15. Frequency components of the F/B sway signal for the pre-, post-exposures and at sickness onset instances. 
(a)

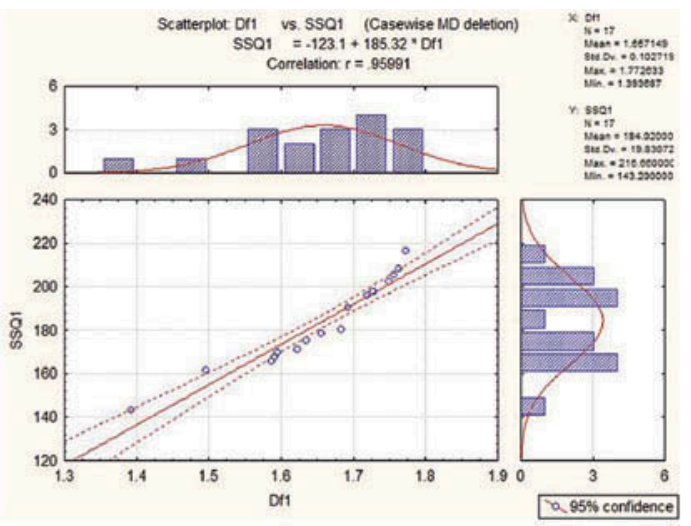

(b)
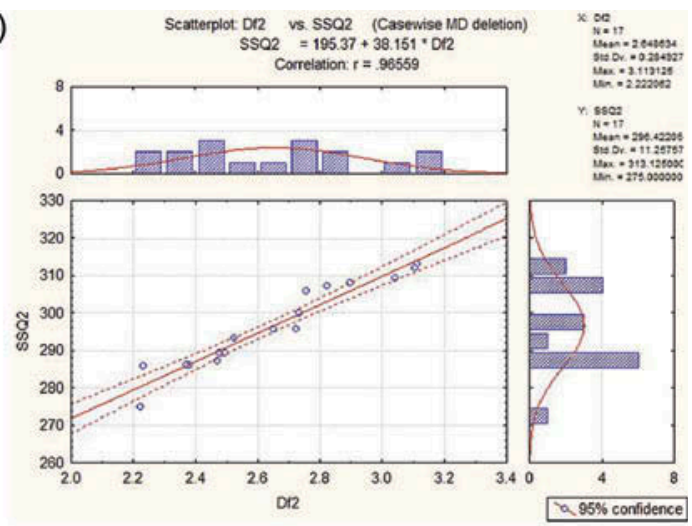

(c)

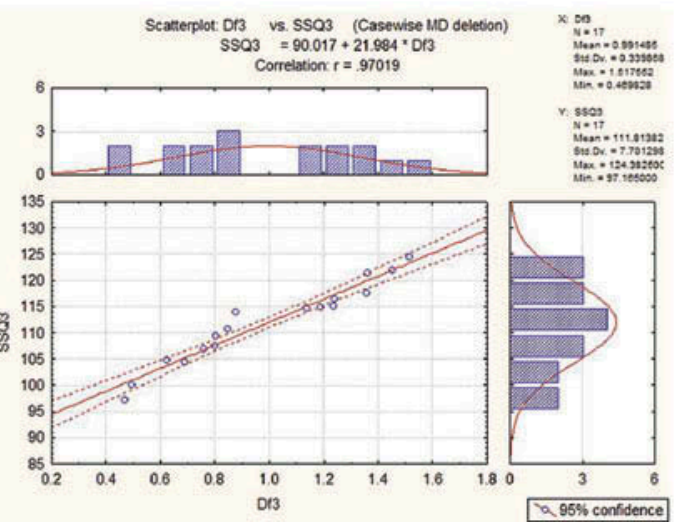

Figure 16. Correlation between the level of sickness and the variation of the frequency components: between (a) $\mathrm{SSQ}_{1}$ and $\Delta \mathrm{f}_{1}$, (b) $\mathrm{SSQ}_{2}$ and $\Delta \mathrm{f}_{2}$, and (c) $\mathrm{SSQ}_{3}$ and $\Delta \mathrm{f}_{3}$.

$M=296.42$ ). Thus, the difference between the LF and HF components is a very effective feature for detecting a VIMS occurrence and estimating the amount of sickness. It even better highlights when a user starts feeling sickness, as (1) the body sway's spectrum split into low-frequency components (associated with voluntary movements) and high-frequency components (associated with involuntary movements), and (2) the high-frequency components move away from $1 \mathrm{~Hz}$ as sickness increases. We therefore assessed the simulation and the second hypothesis. The spectrum of the COG's signal and its frequency components are efficient indicators of the level of sickness of the user.

\section{Prediction of VIMS}

From both simulation and experiment results, we observed that the amount of the COG's projection area dilation, its shape, and the difference between the HF-LF components of body sway are efficient indicators of the level of sickness as they contribute to estimate VIMS. From these results, we can deduce a method to predict the onset of VIMS and track the level of sickness during navigation in an immersive VE. When the level of sickness reaches a certain threshold, the user is informed and the process can be stopped. Figure 17 shows a prediction system that uses the shape, the area, and the

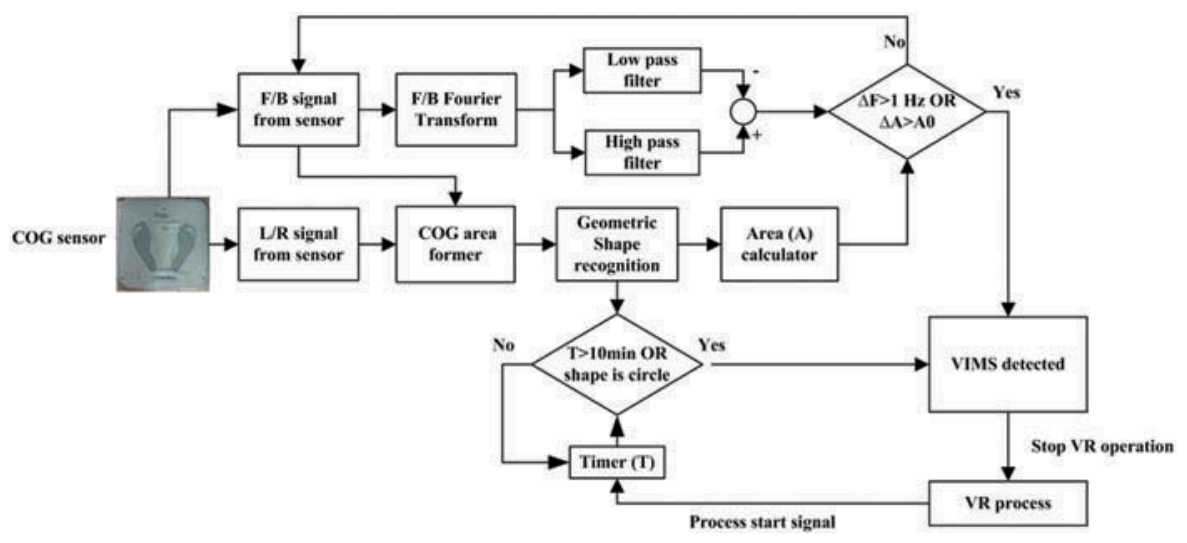

Figure 17. VIMS prediction in a real-time VR application. 
frequency components of the COG to predict an occurrence of VIMS.

In this prediction system, we put another condition for stopping the process, which is not to exceed 10 min inside the VR system as past research showed that sickness can appear beyond this time because of accumulation effect (Oman, 1990). This limit is not necessary, but we keep it just for safety reasons.

\section{Discussion and conclusion}

Much research has been done over the last decades to better understand the reasons of occurring sickness when a human is exposed to motion, especially in the aviation field where pilots should be first trained in a flight simulator before maneuvering real aircraft (Cheung et al., 1991; Ebenholtz, Cohen, \& Linder, 1994; Hosman \& Stassen, 1999; Kellogg, Kennedy, \& Graybiel, 1964). Many of the proposed methods to evaluate sickness have been designed for this field (DiZio \& Lackner, 1998; Kennedy, Fowlkes, \& Lilienthal, 1993b; Kennedy et al., 1993a). However, since the development of VR, these methods have been extensively used as it has been observed that a VR user gets sick when immersed in a $3 \mathrm{D}$ VE. Especially, with the recent increase of interest in using $\mathrm{VR}$, both in industry and in daily life, due to the commercialization of much cheaper devices, focusing on cybersickness has become of primary concern to ensure success of VR facilities.

This study focused on a method to better monitor the level of sickness in a VR application. We considered the body sway as an efficient feature to indicate the occurrence of sickness, in conjunction with SSQs. Past research has shown that the measure of the projection of the COG would be a useful index of the level of sickness (Kennedy \& Stanney, 1996; Stoffregen et al., 2000; Takada et al., 2007). It is also one of the least constraining methods for a VR user compared to other physiological methods. However, just analyzing the sway in the time domain, either using a balance board or a camera, may not be enough as some features to characterize VIMS may be hidden. In particular, voluntary (corresponding to movements controlled by a person) versus involuntary (corresponding to movements non-controlled by the person, indicating potential sickness) movements are hardly distinguishable.

Therefore, we proposed to use not only the area of the COG's projection, studied in most past studies, but also the variation of its shape and its frequency components. We conducted simulation with theoretical stable and marginally stable body movements. We showed that not only the area of the COG's projection dilates and distributes less densely by time, but also its shape changes from an ellipse to a circle. User studies proved that the change in the shape occurs at sickness onset, i.e., when the sickness scores reported by a participant through the SSQs suddenly increase.

To be sure this change in the area's shape is correlated with the occurrence of sickness, we analyzed the body sway signal in the frequency domain. Past research showed that the frequency-domain analysis would be a useful index to distinguish between voluntary and involuntary movements (Bos,
2003). Both simulation and user studies performed in this work showed that without any presence of sickness the frequency components span below $1 \mathrm{~Hz}$; in other words, only voluntary movements occurs. Whereas when a participant starts reporting sickness through the SSQs, i.e., at sickness onset, some frequency components appear above $1 \mathrm{~Hz}$, meaning the body movements become involuntary. The difference between high-frequency components (above $1 \mathrm{~Hz}$ ) and lowfrequency components (below $1 \mathrm{~Hz}$ ) grows by time of navigation in a $3 \mathrm{D} V E$ : the sicker the participants, the farther from $1 \mathrm{~Hz}$ the frequency components.

In this regard, our study confirms findings of previous research, as well as it highlights other features that were hardly explored before (and so augments previous research), that can be used to better monitor sickness in a VR application.

From a modeling perspective of VIMS, when users are navigating a $3 \mathrm{D} \mathrm{VE}$, the conflict between proprioceptive data and the measurement of the sensory organs leads to an overestimation or an underestimation of the Kalman gain $\mathbf{K}$ and the $\mathbf{S}$ matrix (see the model adapted from Oman 1990 in Figure 1). This miscalculation leads to more divergence between actual and estimated sensory data, which in turns affects the sensorimotor command $\mathbf{C}$ and results in less stance posture stability. This miscalculation is however sometimes dissolved by adaptation or habituation in the environment: repeated exposure and adaptation can minimize sickness effects (Money, 1972).

Though we proposed a method to estimate and predict VIMS in a VR application, this prediction is not fully real time as measurements have to be done regularly, i.e., the current task has to be interrupted several times. Indeed, in this work, we were not sure involuntary biases would not have been introduced because of other user's movements (e.g., the user moving his/her head while navigating). Future work will investigate the possibility of monitoring in real time the frequency components of the body sway signal, i.e., without any interruption of the current task.

Note that in our study there were few females compared to males. From past research, it is known that females are generally more prone to sickness (e.g., Koslucher, Munafo, \& Stoffregen, 2016), therefore gender could be an issue in the interpretation of our results. However, in future work, we want to consider having a navigation system with close-loop feedback, namely looping real-time behavioral measurements with navigation control laws so that the navigation system automatically adapts to the level of sickness of the user. Typically when a user starts showing signs of sickness through continuous measurements, the navigation system adapts the navigation speed accordingly to limit the increase of sickness. Thus, our system would be user-dependent but not gender-dependent.

In this study, we focused only on translational movements, but future studies will investigate also rotational movements, as well as other parameters influencing sickness, such as the navigation speed, the system latency, and so on.

\section{Funding}

This research was supported by the French Minister of Economy, Finances and Industry, under contract FUI Callisto. 


\section{ORCID}

Jean-Rémy Chardonnet (D) http://orcid.org/0000-0002-8926-1359

Frédéric Mérienne (iD) http://orcid.org/0000-0003-4466-4776

\section{References}

Bos, J. E. (2003). Method for the prevention of motion sickness, and apparatus for detecting and signaling potentially sickening motions. Patent PCT/NL2003/000119.

Bos, J. E., Bles, W., \& Groen, E. L. (2008). A theory on visually induced motion sickness. Displays, 29 (2), 47-57. doi:10.1016/j. displa.2007.09.002

Cheung, B. S., Howard, I. P., \& Money, K. E. (1991). Visually-induced sickness in normal and bilaterally labyrinthine-defective subjects. Aviation, Space, and Environmental Medicine, 62 (6), 527-531.

Cheung, B. S. K., Howard, I. P., Nedzelski, J. M., \& Landolt, J. P. (1989). Circularvection about earth-horizontal axes in bilateral labyrinthinedefective subjects. Acta Oto- Laryngologica, 108 (5-6), 336-344. doi:10.3109/00016488909125537

Cobb, S. (1999). Measurement of postural stability before and after immersion in a virtual environment. Applied Ergonomics, 30 (1), 4757. doi:10.1016/S0003-6870(98)00038-6

Darken, R. P., \& Sibert, J. L. (1996). Navigating large virtual spaces. International Journal of Human-Computer Interaction, 8 (1), 49-71. doi:10.1080/10447319609526140

Diels, C. (2008). Visually induced motion sickness ( $\mathrm{PhD}$ thesis). Loughborough, UK: Loughborough University.

DiZio, P., \& Lackner, J. R. (1998). Alleviation of motion sickness and postural instability during and after virtual environment exposure. Technical report (TR- 98-009). Orlando, FL: Naval Air Warfare Center Training Systems Division.

Ebenholtz, S. M., Cohen, M. M., \& Linder, B. J. (1994). The possible role of nystagmus in motion sickness: A hypothesis. Aviation, Space, and Environmental Medicine, 65 (11), 1032-1035.

Hettinger, L. J., \& Riccio, G. E. (1992). Visually induced motion sickness in virtual environments. Presence: Teleoperators and Virtual Environments, 1 (3), 306-310. doi:10.1162/pres.1992.1.3.306

Himi, N., Koga, T., Nakamura, E., Kobashi, M., Yamane, M., \& Tsujioka, K. (2004). Differences in autonomic responses between subjects with and without nausea while watching an irregularly oscillating video. Autonomic Neuroscience, 116 (1), 46-53. doi:10.1016/j. autneu.2004.08.008

Holmes, S. R., \& Griffin, M. J. (2001). Correlation between heart rate and the severity of motion sickness caused by optokinetic stimulation. Journal of Psychophysiology, 15 (1), 35-42. doi:10.1027/0269-8803.15.1.35

Hosman, R., \& Stassen, H. (1999). Pilot's perception in the control of aircraft motions. Control Engineering Practice, 7 (11), 1421-1428. doi:10.1016/S0967-0661(99)00111-2

Irwin, J. A. (1881). The pathology of sea-sickness. The Lancet, 118 (3039), 907-909. doi:10.1016/S0140-6736(02)38129-7

James, W. (1881). Sense of dizziness in deaf-mutes. Mind, 23, 412-413. doi:10.1093/mind/os-VI.23.412

Johnson, W. H., Sunahara, F. A., \& Landolt, J. P. (1999). Importance of the vestibular system in visually induced nausea and self-vection. Journal of Vestibular Research, 9 (2), 83-87.

Kaga, K. (1992). Memaino Kouzo: Structure of vertigo (Vol. 1, pp. 23-26). Kanehara, Tokyo: Kanehara Shuppan Co.

Kalman, R. E. (1960). A new approach to linear filtering and prediction problems. Journal of Basic Engineering, 82 (1), 35-45. doi:10.1115/ 1.3662552

Kellogg, R. S., Kennedy, R. S., \& Graybiel, A. (1964). Motion sickness symptomatology of labyrinthine defective and normal subjects during zero gravity maneuvers. Technical report, DTIC Document. WrightPatterson Air Force Base, Ohio, Aerospace Medical Research Laboratories.

Kemeny, A. (2014, April). From driving simulation to virtual reality. Proceedings of Virtual Reality International Conference, Laval, France.

Kemeny, A., Colombet, F., Denoual, T. (2015, March). How to avoid simulation sickness in virtual environments during user displacement.
Proceedings of conference: IS\&T/SPIE Electronic Imaging, The Engineering Reality of Virtual Reality, volume 9392, San Francisco, CA.

Kennedy, R., \& Stanney, K. (1996). Postural instability induced by virtual reality exposure: Development of a certification protocol. International Journal of Human-Computer Interaction, 8 (1), 25-47. doi:10.1080/10447319609526139

Kennedy, R. S., Drexler, J. M., Compton, D. E., Stanney, K. M., Lanham, D. S., \& Harm, D. L. (2003). Configural scoring of simulator sickness, cybersickness and space adaptation syndrome: Similarities and differences. In L. J. Hettinger \& M. W. Haas (Eds), Virtual and adaptive environments: Applications, implications, and human performance (pp. 247-278). Mahwah, NJ: Lawrence Erlbaum Associates.

Kennedy, R. S., Fowlkes, J. E., \& Lilienthal, M. G. (1993b). Postural and performance changes following exposures to flight simulators. Aviation, Space, and Environmental Medicine, 64 (10), 912-920.

Kennedy, R. S., Graybiel, A., McDonough, R. C., \& Beckwith, D. (1968). Symptomatology under storm conditions in the North Atlantic in control subjects and in persons with bilateral labyrinthine defects. Acta Oto-Laryngologica, 66 (1-6), 533-540. doi:10.3109/ 00016486809126317

Kennedy, R. S., Lane, N. E., Berbaum, K. S., \& Lilienthal, M. G. (1993a). Simulator sickness questionnaire: An enhanced method for quantifying simulator sickness. The International Journal of Aviation Psychology, 3 (3), 203-220. doi:10.1207/s15327108ijap0303_3

Khalil, H. K. (2002). Nonlinear systems (Vol. 3). Upper Saddle River, NJ: Prentice hall.

Kolasinski, E. M. (1995). Simulator Sickness in Virtual Environments, (1027). Technical report. Alexandria, VA: U.S. Army Research Institute.

Koslucher, F., Munafo, J., \& Stoffregen, T. A. (2016). Postural sway in men and women during nauseogenic motion of the illuminated environment. Experimental Brain Research, 234 (9), 2709-2720. doi:10.1007/s00221-016-4675-8

Loram, I. D., \& Lakie, M. (2002). Direct measurement of human ankle stiffness during quiet standing: The intrinsic mechanical stiffness is insufficient for stability. The Journal of Physiology, 545 (3), 1041-1053. doi:10.1113/jphysiol.2002.025049

Masani, K., Popovic, M. R., Nakazawa, K., Kouzaki, M., \& Nozaki, D. (2003). Importance of body sway velocity information in controlling ankle extensor activities during quiet stance. Journal of Neurophysiology, 90 (6), 3774-3782. doi:10.1152/jn.00730.2002

MathWorks (2015). MATLAB Simulink, R2015b release. MathWorks.

Money, K. E. (1972). Measurement of susceptibility to motion sickness. In AGARD Conference Proceedings (pp. B2-1-B2-4), Neuilly-sur-Seine, France.

Morasso, P. G., \& Schieppati, M. (1999). Can muscle stiffness alone stabilize upright standing? Journal of Neurophysiology, 82 (3), 1622-1626.

Murata, A. (2004). Effects of duration of immersion in a virtual reality environment on postural stability. International Journal of HumanComputer Interaction, 17 (4), 463-477. doi:10.1207/ s15327590ijhc1704_2

Okawa, T., Tokita, T., Shibata, Y., Ogawa, T., \& Miyata, H. (1995). Stabilometry. Significance of locus length per unit area (L/A) in patients with equilibrium disturbances. Equilibrium Research, 55 (3), 283-293. doi:10.3757/jser.55.283

Oman, C. M. (1990). Motion sickness: A synthesis and evaluation of the sensory conflict theory. Canadian Journal of Physiology and Pharmacology, 68 (2), 294-303. doi:10.1139/y90-044

Reason, J. T. (1978). Motion sickness adaptation: A neural mismatch model. Journal of the Royal Society of Medicine, 71 (11), 819-829.

Reason, J. T., \& Brand, J. J. (1975). Motion sickness. London, England: Academic Press.

Riccio, G. E., \& Stoffregen, T. A. (1991). An ecological theory of motion sickness and postural instability. Ecological Psychology, 3 (3), 195-240. doi:10.1207/s15326969eco0303 2

Rolnick, A., \& Lubow, R. E. (1991). Why is the driver rarely motion sick? The role of controllability in motion sickness. Ergonomics, 34 (7), 867-879. doi:10.1080/00140139108964831

Sharples, S., Cobb, S., Moody, A., \& Wilson, J. R. (2008). Virtual reality induced symptoms and effects (VRISE): Comparison of head 
mounted display (HMD), desktop and projection display systems. Displays, 29 (2), 58-69. doi:10.1016/j.displa.2007.09.005

Smart, L. J., Stoffregen, T. A., \& Bardy, B. G. (2002). Visually induced motion sickness predicted by postural instability. Human Factors: The Journal of the Human Factors and Ergonomics Society, 44 (3), 451-465. doi: $10.1518 / 0018720024497745$

Smith, J. W. (1957). The forces operating at the human ankle joint during standing. Journal of Anatomy, 91 (Pt4), 545-564.

Stanney, K. M., \& Hash, P. (1998). Locus of user-initiated control in virtual environments: Influences on cybersickness. Presence: Teleoperators and Virtual Environments, 7 (5), 447-459. doi:10.1162/ 105474698565848

Stoffregen, T. A., Hettinger, L. J., Haas, M. W., Roe, M. M., \& Smart, L. J. (2000). Postural instability and motion sickness in a fixed-base flight simulator. Human Factors: The Journal of the Human Factors and Ergonomics Society, 42 (3), 458-469. doi:10.1518/ 001872000779698097

Takada, H., Fujikake, K., Miyao, M., \& Matsuura, Y. (2007, December), Indices to detect visually induced motion sickness using stabilometry. Presented at First International Symposium on Visually Induced Motion Sickness, Fatigue, and Photosensitive Epileptic Seizures (VIMS2007) (pp. 178-183), Hong Kong, China.

TechnoConcept. (2007). Techno-concept balance board manual, Notice Logiciel SabotSoft. France: TechnoConcept.

Yokota, Y., Aoki, M., Mizuta, K., Ito, Y., \& Isu, N. (2005). Motion sickness susceptibility associated with visually induced postural instability and cardiac autonomic responses in healthy subjects. Acta Oto-Laryngologica, 125 (3), 280-285. doi:10.1080/00016480510003192

\section{About the Authors}

Jean-Rémy Chardonnet received his Ph.D. degree in robotics from the Université de Montpellier II, France, in 2009. He was then with INRIA Grenoble, France. He is currently Assistant Professor at Arts et Métiers and CNRS Le2i Institut Image, Chalon-sur-Saône, France. His research interests include virtual reality and multimodal interaction.

Mohammad Ali Mirzaei received his Ph.D. degree in virtual reality from Arts et Métiers, France in 2014. His research focuses on signal/image processing and real-time systems.

Frédéric Mérienne is Full Professor at Arts et Métiers ParisTech and head of the research team in Virtual Immersion of the Le2i laboratory (Bourgogne Franche-Comté University, Arts et Métiers, CNRS). His research topics concern multisensory immersive techniques for interacting with a digital mock-up.

\section{Appendix}

\section{Q1-Examinee General Information (Pre-Exposure Questionnaire)}

1. First name

2. Family name

3. Email address

4. Age

5. Weight

6. Gender: $\square$ Man $\square$ Woman

7. Profession: $\square$ Student $\square$ Engineer $\square$ Programmer

a. $\square$ Office clerk $\square$ Other:

8. Experience with a computer:

a. Professional gamers

Level: $\square$ Every day $\square$ At least once a week $\square$ At least once a month

$\square$ Played before a lot but not now

Played but I do not know exactly how much

b. Computer users

Level: $\square$ Daily users (office, clerk, ...)

$\square$ Use the computer not so much

9. From the devices listed below, which one have you used so far:

$\square$ Kinect $\square$ Gamepad $\square$ Flystick

10. Have you played to a laser tag game so far: $\square$ Yes $\square$ No

11. Do you get sick when you travel: $\square$ Yes $\square$ No

12. If the answer to item 11 is yes, which of the following travel vehicles makes you sickest: $\square$ Car $\square$ Train $\square$ Airplane

$\square$ Boat $\square$ Ship/ferry

13. When the traveling vehicle is in motion, do you study: $\square$ Yes $\square$ No

14. If the answer to item 13 is yes, do you feel (during study): $\square$ Headache $\square$ Eyestrain $\square$ Fatigue

15. If you are a woman, have you been pregnant: $\square$ Yes $\quad \square$ No

16. Do you have any auditorial disorder: $\square$ Yes $\square$ No

17. If the answer to item 16 is yes, what is the level of disorder:

$\square$ Slight $\square$ Moderate $\square$ Headphone implanted

$\square$ Severe $\square$ Near to deaf

18. Do you have any visual disorder: $\square$ Yes $\square$ No

19. If the answer to item 18 is yes, what is the level of disorder:

$\square$ Slight $\square$ Moderate $\square$ Severe $\square$ Other

20. Do you wear glasses: $\square$ Yes $\square$ No 